\title{
Electro-clinical characteristics and postoperative outcome of medically refractory tumoral temporal lobe epilepsy
}

\author{
Samhita Panda, Venkataraman V. Radhakrishnan, Kurupath Radhakrishnan, Ravi M. Rao, \\ Sankara P. Sarma
}

Department of Neurology, R. Madhavan Nayar Center for Comprehensive Epilepsy Care, Sree Chitra Tirunal Institute for Medical Sciences and Technology, Trivandrum, India

\begin{abstract}
Background: Very few studies have specifically addressed surgical treatment and outcome of patients with tumor-related temporal lobe epilepsy (TLE). Aim: To define the postoperative seizure outcome and the factors that influenced the outcome of patients with tumor-related TLE. Materials and Methods: We selected patients whose surgical pathology revealed a temporal lobe neoplasm and who had completed $\geq 1$ year of postoperative follow-up. We reviewed the clinical, EEG, radiological and pathological data, and the seizure outcome of these patients and assessed the factors that influenced the outcome. Results: Out of the 409 patients who underwent surgery for refractory TLE during the 8 -year study period, there were $34(8.3 \%)$ patients with temporal lobe neoplasms. The median age at surgery was 20 years and the median duration of epilepsy prior to surgery was 9.0 years. MRI revealed tumor in the mesial location in $21(61.8 \%)$ patients. Interictal and ictal epileptiform EEG abnormalities were localized to the side of th lesion in the majority. Mesial temporal lobe structures were included in the resection, if they were involved by the tumor; otherwise, lesionectomy alone was performed. During a median follow-up of 4 years, $27(79 \%)$ patients were completely seizure-free. The only factor that predicted long-term seizurefree outcome was being seizure-free during the first two postoperative years. Conclusions: Our results emphasize the fact that in patients with tumoral TLE, when the seizures are medically refractory, surgery offers potential for cure of epilepsy in the majority.
\end{abstract}

Key Words: Epilepsy, temporal lobe, tumoral epilepsy, medically refractory epilepsy

\section{Introduction}

More than two-thirds of carefully selected patients with medically refractory temporal lobe epilepsy (TLE) become seizure-free following surgery. ${ }^{[1,2]} \mathrm{A}$ magnetic resonance imaging (MRI)-detected lesion is one of the most important factors that predicts the seizure outcome following epilepsy surgery. ${ }^{[2,3]}$ Although emphasis is often placed on mesial temporal sclerosis-associated TLE, ${ }^{[1-3]}$ neuropathological series of surgically treated TLE have reported neoplasms in $10 \%$ to $30 \%$ of the specimens. ${ }^{[4,5]}$

The histopathological profile of tumor-related chronic epilepsy is quite different from the usual pattern of primary brain neoplasms. ${ }^{[4,5]}$ Although low-grade tumors such as ganglioglioma and dysembryoplastic neuroepithelial tumor (DNET) account for only a very small proportion of primary brain neoplasms, they are the lesions frequently associated with tumoral refractory epilepsy..$^{[4,5]}$

Several studies have addressed the surgical treatment and outcome of patients with tumoral chronic epilepsy. ${ }^{[6-14]} \mathrm{A}$ majority of them have either combined a variety of neoplasms involving different brain regions, ${ }^{[9,11]}$ or have assembled a specific tumor type involving different brain locations. ${ }^{[7,8]}$ Tumorrelated refractory epilepsy encompasses a heterogeneous group of temporal and extratemporal epilepsies with different pathological substrates and postoperative seizure outcomes. ${ }^{[5]}$ To our knowledge, very few studies have specifically addressed the surgical treatment and outcome of tumoral TLE. ${ }^{[10,12-14]}$ This is all the more surprising because the temporal lobe is the commonest site of the neoplasms associated with medically refractory epilepsy. ${ }^{[5]}$ A literature survey from the Indian subcontinent revealed no published reports which highlighted the electro-clinical profile and postoperative outcome of tumoral chronic TLE.

With the above considerations, we undertook this study to review the clinical, electroencephalographic (EEG), and radiological data of patients with tumoral TLE, who had undergone evaluation and surgery through our epilepsy program, 
and to define the postoperative seizure outcome of these patients and the factors that influenced the outcome.

\section{Materials and Methods}

Between March 1995 through March 2003, 409 patients underwent surgery for medically refractory TLE at our epilepsy center. The details of our presurgical evaluation protocol, which includes history, optimum 1.5T MRI, interictal scalp EEG, video-EEG monitoring with scalp/sphenoidal electrodes, and neuropsychological assessment, have been described previously. ${ }^{[15]}$ None of the patients were subjected to positron emission tomography and single photon emission computed tomography or invasive EEG studies. For the purpose of this study, we selected patients whose surgical pathology revealed a temporal lobe neoplasm and who had completed $\geq 1$ year of postoperative follow-up. We excluded patients in whom the indication for surgery primarily was the lesion and not refractory epilepsy.

We reviewed all the preoperative MRI films to delineate the site, size, and extent of the tumor, as well as the contrast enhancement, calcification, cystic changes and mass effect, and associated hippocampal atrophy and sclerosis. We used collateral sulcus as the boundary between the medial and lateral location of the tumor in the temporal lobe.

Standard 10-20 system of extracranial electrode placement was used with additional anterior temporal (T1 and T2) electrodes. The distribution of interictal epileptiform discharges (IEDs) during prolonged video-EEG monitoring was assessed by visual analysis of interictal EEG samples of 15 seconds every 15 minutes. We classified scalp recorded ictal EEG activity into three patterns: regular, well-modulated, 5 - $9 \mathrm{~Hz}$, unilateral temporal and/or subtemporal rhythm (Type 1), polymorphic slower $(<5 \mathrm{~Hz}$ ) rhythm with broader unihemispheric distribution (Type 2), and diffuse irregular slowing in the background activity (Type 3). ${ }^{[16,17]}$ The diagnosis of the videoEEG recorded seizures as temporal was based on the well recognized semiological characteristics (such as epigastric aura, behavioral arrest, oro-alimentary and limb automatisms, dystonic limb posturing, ictal speech, and post-ictal dysphasia) along with the localization and pattern of ictal EEG discharges. ${ }^{[17,18]}$

\section{Surgical procedures}

All surgeries were undertaken under general anesthesia. The operative procedure was tailored as warranted by the site and extent of the tumor: Intraoperative electrocorticography (ECoG) was performed during all resections undertaken after September 2001.

\section{Pathological examination}

Four-micrometer-thick histological sections were generated from $10 \%$ formalin-fixed, paraffin-embedded tissue and stained with hematoxylin and eosin. ${ }^{[19]}$ For pathological diagnosis, we used the WHO definition of primary brain neoplasms. ${ }^{[20]}$ While the histopathological diagnosis of ganglioglioma required an intimate mixture of neoplastic ganglion cells and neoplastic astrocytes, cystic spaces with floating neurons and oligodendroglia-like cells characterized DNET. We defined associated mesial temporal sclerosis as the loss of neuronal cell population of $\geq 30 \%$ in the CA1 sector of the hippocampal formation with or without neuronal loss and gliosis involving other mesial temporal lobe structures. ${ }^{[19]}$
Patients were regularly followed up at 3 months and 12 months after surgery and at yearly intervals thereafter. The postoperative seizure outcome at last follow-up was defined as seizure-free without antiepileptic drugs, seizure-free with antiepileptic drugs, and unfavorable outcome (seizure recurrence of any type). ${ }^{[15]}$ Neuropsychological assessment and EEG were repeated according to standard protocol during each follow-up visit until 3 years. ${ }^{[15,21]}$ Postoperative MRI was undertaken only in those with seizure recurrence.

\section{Statistical methods}

We analyzed the data using the SPSS statistical package. We tested the association of different clinical, EEG and MRI characteristics with the seizure outcome at last follow-up utilizing 2-tailed t-test, Pearson's chi-square test and Fisher's exact test, as appropriate. We used standard deviation (SD) to define the dispersion.

\section{Results}

\section{Patient characteristics}

Out of the 409 patients who underwent surgery for refractory TLE during the study period, there were 34 (8.3\%) patients (22 males, 12 females) with temporal lobe neoplasms. The demographic and clinical features of these patients are provided in Table 1 . The median age at surgery was 20.0 years (range 1.3 to 37 years), and the median duration of epilepsy prior to surgery was 9.0 (range 1 to 25 ) years. The antecedents included family history of seizures (3 patients), febrile seizures (5 patients) and encephalitis (1 patient). Seizure semiology suggested complex partial seizures of temporal lobe origin in all patients; in addition, secondarily generalized seizures occurred in 21 patients. Four of our patients exhibited preoperative overt psychiatric disturbances (depression with suicidal ideation, obsessive compulsive disorder in one each and conduct disorder in 2 patients).

\section{Neuroimaging findings}

Computed tomography (CT) scan was available in 10 patients, in 2 of whom CT did not identify the tumor. MRI defined the location and extent of the tumor in all patients (Table 1). The tumor was confined to the mesial temporal lobe in 21 patients $(61.8 \%)$ and to the lateral temporal lobe in 9 patients, while in the remaining 4 , the lesion involved both the mesial and lateral temporal regions. The MRI findings included cystic changes in the tumor in 15, contrast enhancement in 11, mass-effect in 6 , and perilesional edema in 2 patients. Calcification in the CT and/or MRI was noted in 11 patients. Concomitant mesial temporal sclerosis was observed in 3 patients.

\section{EEG data}

Temporal IEDs were present exclusively on the side of the lesion in 24 patients. Bilateral independent temporal IEDs were seen in 7 patients. While 1 patient had extratemporal IEDs, 2 patients did not manifest any IEDs. During video- 


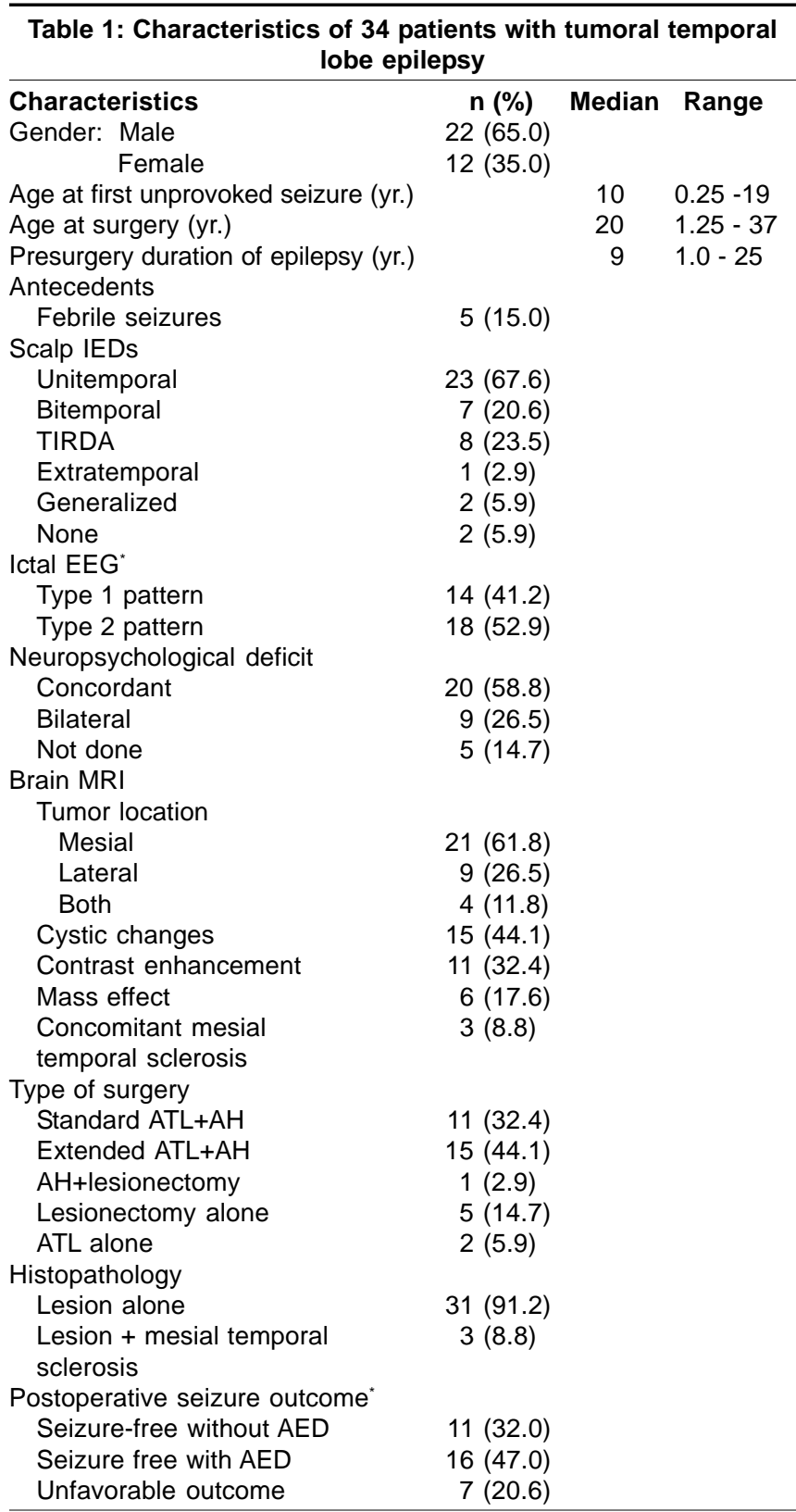

IEDs - interictal epileptiform discharges; AED - antiepileptic drugs; ATL - anterior temporal lobectomy; $\mathrm{AH}$ - amygdalohippocampectomy; TIRDA - temporal intermittent rhythmic delta activity; 'See text for definitions

EEG monitoring, temporal intermittent rhythmic delta activity (TIRDA) ipsilateral to the tumor location was seen in 8 patients. Ictal EEG pattern was Type 2 in 18 patients and Type 1 in 14 patients (Table 1). No seizures were recorded in 2 patients. The mean duration of the video-EEG monitoring was 2.9 days (range 6 hours to 9 days).

\section{Surgery}

Table 1 lists the surgical procedures employed. While lesionectomy alone was sufficient in 5 patients, involvement of the mesial structures by the tumor necessitated either anterior temporal lobectomy with amygdalohippocampectomy (21 patients) or selective amygdalohippocampectomy (1 patient).
None underwent postoperative radiotherapy. Two patients with postoperative seizure recurrence required repeat surgery to resect residual lesions.

\section{Pathology}

The distribution of patients according to pathological diagnosis is provided in Figure 1. Ganglioglioma (Figure 2) was the commonest tumor type (23 patients). Low-grade gliomas and DNET (Figure 3) were diagnosed in 8 and 3 patients, respec-

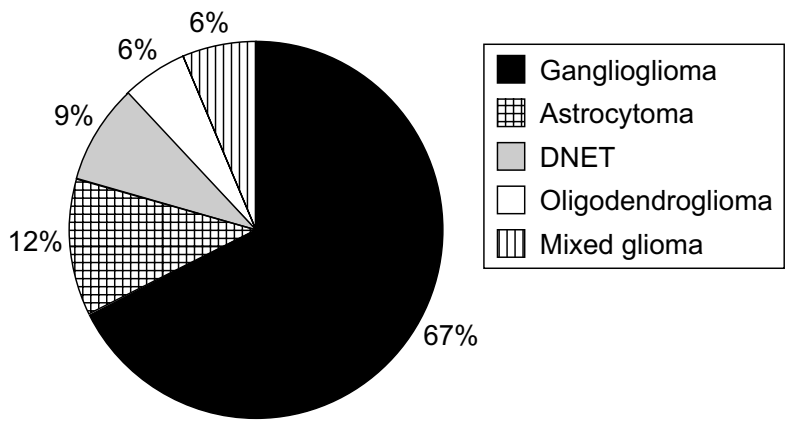

Figure 1: Distribution of patients according to tumor type

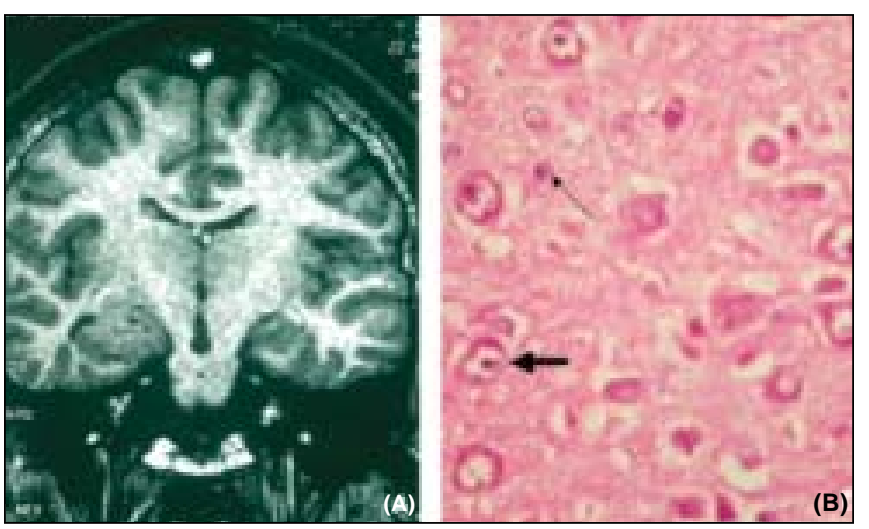

Figure 2: (A) T1-weighted coronal MRI sequence from one of our patients shows a neoplasm involving the right mesial temporal region,

(B) photomicrograph shows admixture of neoplastic ganglion cells (thick arrow) and astrocytic cells (thin arrow) characteristic of ganglioglioma (H/E x200)

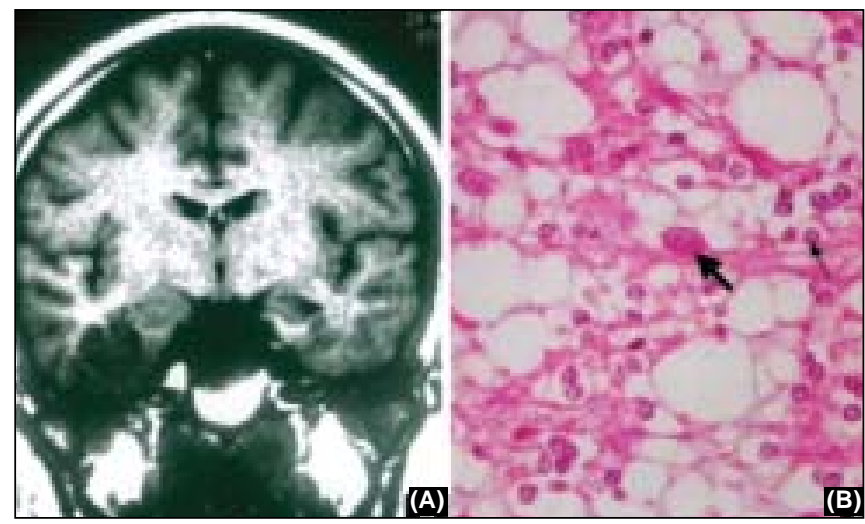

Figure 3: (A) T1-weighted coronal MRI sequence from one of our patients shows right temporal lobe neoplasm with cystic changes, (B) photomicrograph shows microcystic spaces with floating neurons (thick arrow) and oligodendrocyte-like cells (thin arrow) characteristic of dysembryoplastic neuroepithelial tumor (H/E x200) 
tively. The interesting association of DNET with melanotic differentiation has been reported previously in 1 of our patients. ${ }^{[22]}$

\section{Seizure outcome}

During the median duration of follow-up of 4 (range 1-9) years, 27 of 34 patients $(79.0 \%)$ were completely seizure-free at last follow-up; antiepileptic drugs could be successfully discontinued in $11(32 \%)$ of them (Table 1$)$. The seizure-free outcome at 2 years $(23 / 29,79.3 \%)$ and at 5 years $(9 / 12,75 \%)$ did not differ $(P=1.000)$. Seven patients continued to have postoperative seizures, although 5 of them experienced $>75 \%$ seizure reduction. Two patients continued to have auras, which did not impair the activities of daily living.

\section{Complications}

No patient died. Long-term morbidity in the form of hemiparesis and hemianopia occurred in 3 patients each. Fifteen patients had quadrantinopic field defect, which did not bother them. None of our patients exhibited any significant postoperative aggravation of psychiatric disturbances. Residual lesion was detected in 5 out of 8 patients who underwent postoperative MRI.

\section{Predictors of outcome}

The attributes of the patients with excellent and unfavorable seizure outcomes are compared in Table 2. Age at surgery, duration of epilepsy prior to surgery, preoperative interictal and ictal EEG data, distribution of neuropsychological defi- cit and histopathological tumor type did not influence the outcome. While 25 of $27,(92.6 \%)$ patients who were seizurefree at last follow-up did not have seizures in the first postoperative year, only 3 of $7(42.9 \%)$ with seizure recurrence were seizure-free during the first postoperative year. The difference $(49.7 \%, 95 \%$ confidence interval $11.7 \%$ - $87.7 \%$ ) was significant $(P=0.01)$. The difference was even more significant for those who remained seizure-free for two years $(67.7 \%$, $33.5 \%-99.1 \%, P=<0.001)$. The seizure outcome of those with tumor only $(25 / 32,80.6 \%)$ and of those with tumor plus mesial temporal sclerosis (dual pathology) $(2 / 3,66.7 \%$ ) did not differ $(P=0.511)$. Similarly, there was no significant difference $(P=0.317)$ in the seizure outcome between the patients who underwent standard anterior temporal lobectomy (10/ $11,90.9 \%)$, extended anterior temporal lobectomy (10/15, $66.7 \%)$ or lesionectomy $(5 / 6,83.3 \%)$. Tumor location, whether mesial $(16 / 21,76.2 \%)$ or lateral $(7 / 9,77.8 \%)$, did not influence the seizure outcome $(P=1.000)$. Seizure-free outcome was seen in all 3 patients with post-resection IEDs $(100 \%)$ compared to 5 of 8 patients with no post-resection IEDs $(62.5 \%)$ in the ECoG $(P=0.49)$.

\section{Discussion}

Tumor-related epilepsy constitutes about one-third of patients with medically refractory epilepsy. ${ }^{[4,5]}$ A number of studies have investigated the clinical features, electrographic cor-

Table 2: Comparison of the attributes of patients with seizure-free and unfavorable seizure outcomes

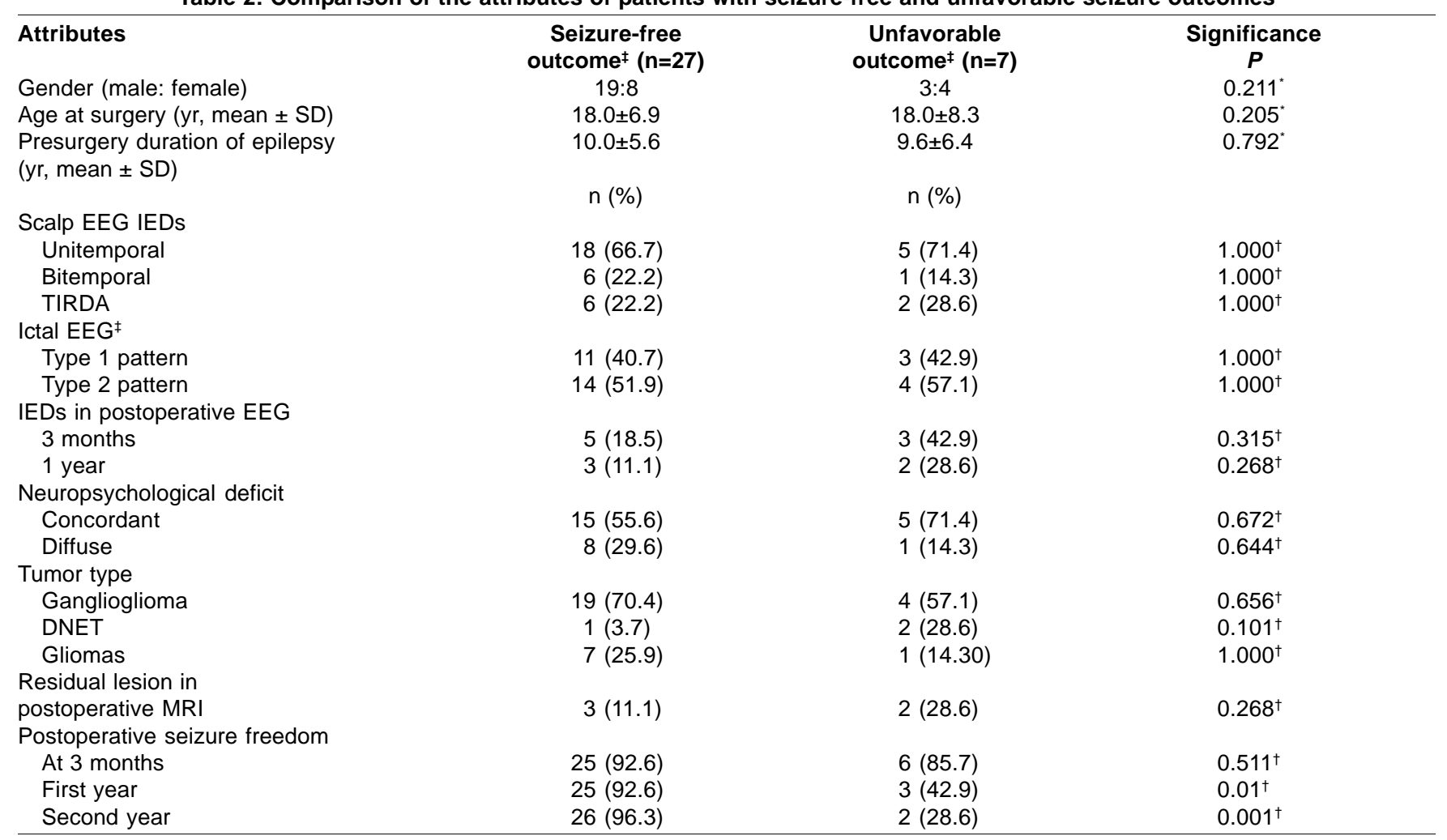

IEDs - interictal epileptiform abnormalities, SD - standard deviation, DNET - dysembryoplastic neuroepithelial tumor, TIRDA - temporal intermittent rhythmic delta activity; ' $P$ by Student's $t$ test, ${ }^{\dagger} P$ by Fisher's exact test; $¥$ See text for definitions 
relates and postoperative seizure outcome of patients with chronic epilepsy due to tumors of different histological types located at different brain regions. ${ }^{[6-14,23]}$ The electro-clinical features, presurgical evaluation strategy, surgical treatment and outcome differ considerably between temporal and extratemporal epilepsies. ${ }^{[5]}$ Only very few studies have specifically addressed the clinical, electrographic and surgical outcome of tumoral TLE, ${ }^{[10,12-14]}$ and none of them are from India. This prompted us to review the clinical, electrophysiological and radiological data, and seizure outcome of patients with tumor-related TLE who had undergone surgery in our Epilepsy Program.

Because of differences in the selection and histopathological criteria, the distribution of the neoplasms according to type has varied widely between series of patients with tumoral chronic epilepsy. ${ }^{[4,5,9,24]}$ Nevertheless, in the majority of the series, as in ours, ganglioglioma is the commonest tumor type, followed by other low-grade gliomas and DNET. ${ }^{[5]}$ A majority of the patients with tumoral refractory epilepsy, as in our series, gets recruited for surgery in early adult life after having had refractory seizures for a decade or more. ${ }^{[5,8,9]}$

Among our 34 patients with tumoral TLE, during a median postoperative follow-up period of 4 years, seizure freedom was achieved in nearly $80 \%$ of patients; one-third of them were completely free of AEDs. Over $90 \%$ of our patients had more than $75 \%$ seizure reduction at last follow-up. Among the 68 patients, who had undergone evaluation and surgery for refractory epilepsy in the Yale University Epilepsy Program, ${ }^{[10]}$ during a mean period of follow-up of 9 years after surgical intervention, $65 \%$ of patients were seizure-free and $87 \%$ had substantial seizure reduction. Our results are in agreement with those from epilepsy surgery centers in the West ${ }^{[10,12-14]}$ and emphasize the fact that in patients with tumoral TLE, when the seizures are medically refractory, surgery offers potential cure of epilepsy in the majority. Although no systematic study has so far been conducted comparing patients with tumors in temporal and extratemporal locations, tumoral TLE is generally considered to have a better seizure-free outcome following surgery. ${ }^{[5]}$

In the Yale University series of patients with tumoral TLE, ${ }^{[10]}$ a better postoperative seizure outcome was found in patients with early surgical intervention and complete tumor resection. However, other studies have failed to find a significant association between age at surgery and postoperative seizure outcome. ${ }^{[25,26]}$ While incomplete tumor resection was an important cause of poor postoperative seizure outcome in some studies, ${ }^{[9,10,27]}$ the presence of residual tumor did not significantly influence the seizure outcome in other studies. ${ }^{[8,28]}$ In the present series of patients with tumoral TLE, our inability to find a significant association of these variables with postoperative seizure outcome may be a reflection of the small number of patients who had an unfavorable outcome. Moreover, considering the financial constraints of the patients and the benign nature of the neoplasms resected, we undertook postoperative MRI only in those with seizure recurrence. Early successful surgical intervention will minimize the adverse medical, behavioral and psychosocial consequences on longstanding refractory epilepsy. ${ }^{[15]}$ The objective of any brain tumor surgery is the complete resection of the lesion, if feasible.$^{[5,29]}$ As we observed, several epilepsy surgery outcome studies have shown that long-term seizure control is best when the patient is seizure-free during the first two years following surgery. ${ }^{[23,30,31]}$

In a comparative analysis of the distribution of the IEDs as seen in the scalp EEG in TLE due to hippocamal sclerosis versus mesial temporal lobe tumors, Hamer et al ${ }^{[32]}$ observed more widespread distribution of IEDs in tumor patients compared to the restricted spiking zone at the anterior temporal region in patients with hippocampal sclerosis. The presence of IEDs over the contralateral temporal lobe did not affect the seizure outcome in our patients. The patterns of scalprecorded ictal EEG patterns have been correlated with the etiological subtypes of TLE. ${ }^{[16,17]}$ Whereas a localized wellmodulated, subtemporal theta frequency rhythm (Type 1) signified hippocampal sclerosis, a polymorphic slower rhythm with broader hemispheric distribution was more often associated with other causes. ${ }^{[16,17]}$ In view of the poor predictive value of the EEG abnormalities in patients with tumor-related TLE, the need of long-term video-EEG monitoring in such patients has been questioned. ${ }^{[8]}$ Ictal monitoring, however, is the only method to document the patient's habitual seizures and to exclude non-epileptic behavioral events. Some studies have found better seizure outcome in patients with tumor who underwent resection of the epileptogenic cortex in addition to the tumor, guided by ECoG. ${ }^{[11,26,33]}$ However, in an analysis of the postoperative seizure outcome among 60 children with tumor-related refractory seizures, favorable seizure outcome was achieved in the majority without the assistance of intraoperative ECoG. ${ }^{[27]}$ Intraoperative ECoG was done in 13 of our patients to guide the tumor resection. There was no difference in the seizure outcome among those operated with and without ECoG.

Although an increased occurrence of psychosis with predominant depressive and paranoid features following surgical treatment of ganglioglioma was initially postulated over 40 years ago ${ }^{[34]}$ recent temporal lobectomy series have failed to substantiate this observation. ${ }^{[35,36]}$ We did not observe major psychosis in the postoperative period in any one of them or in those with other neoplasms. However, the psychiatric disturbances observed preoperatively in 4 patients persisted in the postoperative period without significant aggravation.

Our results are in agreement with prior reports regarding the imaging findings and the optimal surgical strategy ${ }^{[5]} \mathrm{A}$ majority of our patients had removal of the mesial temporal lobe structures because, as suggested by the MRI and intraoperative findings, they were involved by the tumor. When 
the lesion was confined to the lateral temporal region, we did lesionectomy alone. In patients with tumoral TLE, in the absence of tumor invasion of the hippocampus and/or secondary hippocampal sclerosis, inclusion of mesial structures in the resection is unnecessary, as it has not been shown to improve seizure outcome. ${ }^{[5,8,10,37]}$

\section{References}

1. Engel J Jr, Wiebe S, French J, Sperling M, Williamson P, Spencer D, et al Practice parameter: Temporal lobe and localized neocortical resections for epilepsy. Report of the Quality Standards Subcommittee of the American Academy of Neurology, in association with the American Epilepsy Society and the American Association of Neurological Surgeons. Neurology 2003;60:538-47.

2. Radhakrishnan K, So EL, Silbert PL, Jack CR Jr, Cascino GD, Sharbrough FW, et al. Predictors of outcome of anterior temporal lobectomy for intractable epilepsy: A multivariate study. Neurology 1998;51:465-71.

3. MeIntosh AM, Wilson S.J, Berkovic SF. Seizure outcome after temporal lobectomy: Current research practice findings. Epilepsia 2001;42:1288-307.

4. Wolf HK, Wiestler OD. Surgical pathology of chronic epileptic seizure disorders. Brain Pathol 1993;3:371-80.

5. Wetjen NM, Radhakrishnan K, Cohen-Gadol AA, Cascino GD. Resective surgery of neoplastic lesions for epilepsy. In: Shorvon SD, Perucca E, Fish DR, Dodson WE, editors. The Treatment of Epilepsy. Oxford: Blackwell Science; 2004. p. 728-41.

6. Lang FF, Epstein F.J, Ransohoff J, Allen JC, Wisoff J, Abbott IR, et al. Central nervous system gangliogliomas. Part 2: Clinical outcome. J Neurosurg 1993;79:867-73.

7. Celli P, Scarpinati M, Nardacei B, Cervoni L, Cantore GP. Gangliogliomas of the cerebral hemispheres. Report of 14 cases with long-term follow-up and review of literature. Acta Neurochir 1993;125:52-7.

8. Morris HH, Matkovic Z, Estes ML, Prayson RA, Comair YG, Turnbull J, et al. Ganglioglioma and intractable epilepsy: Clinical and neuropsychologic features and predictors of outcome after surgery. Epilepsia 1998;39:307-13.

9. Morris HH, Estes ML, Gilmore R, Van Ness PC, Barnett GH, Turnbull J. Chronic intractable epilepsy as the only symptom of primary brain tumor. Epilepsia 1993;34:1038-43.

10. Zaatreh MM, Firlik KS, Spencer DD, Spencer SS. Temporal lobe tumoral epilepsy. Characteristics and predictors of surgical outcome. Neurology $2003 ; 61: 636-41$.

11. Beger MS, Ghatan S, Haglund MM, Dobbins J, Ojemann GA. Low-grade gliomas associated with intractable epilepsy: Seizure outcome utilizing electrocortcography during tumor resection. J Neurosurg 1993;79:62-9.

12. Hennessy MJ, Elwes RDC, Honavar M, Rabe-Hesketh S, Binnie CD, Polkey CE. Predictors of outcome and pathological considerations in the surgical treatment of intractable epilepsy associated with temporal lobe lesions. J Neurol Neurosurg Psychiatry 2001;70:450-8.

13. Arseni C, Petrovici IN. Epilepsy in temporal lobe tumours. Eur Neurol $1971 ; 5: 201-4$

14. Jorge CL, Nagahashi-Marie SK, Pedreira CC, Rosemberg S, Valerio RM, Valente $\mathrm{KD}$, et al. Clinical characteristies and surgical outcome of patients with temporal lobe tumors and epilepsy. Arq Neuropsiquiatr 2000;58:1002-8.

15. Rao MB, Radhakrishnan K. Is epilepsy surgery possible in countries with limited resources? Epilepsia 2000;41:S31-4.

16. Assaf BA, Ebersole JS. Visual and quantitative ictal EEG predictors of outcome after temporal lobectomy. Epilepsia 1999;40:52-61.
17. Sylaja PN, Radhakrishnan K. The role of scalp EEG in the presurgical evaluation of patients with medically refractory temporal lobe epilepsy. Am J END Technol 2001;41:116-35.

18. Williamson PD, Thadani VM, French JA, Darcy TM, Mattson RH, Spencer SS, et al. Medial temporal lobe epilepsy: Videotape analysis of objective clinical characteristics. Epilepsia 1998;39:1182-8.

19. Radhakrishnan VV, Rao MB, Radhakrishnan K, Thomas SV, Nayak SD, Santoshkumar B, et al. Pathology of temporal lobe epilepsy: An analysis of 100 consecutive surgical specimens from patients with medically refractory epilepsy. Neurol India 1999;47:196-201.

20. Kleihues P, Burger PC, Scheithauer BW. Histological Typing of Tumors of the Central Nervous System, $2^{\text {nd }}$ Ed., Berlin: Springer Verlag; 1993.

21. Alexander A, Thomas SV, Radhakrishnan K. The role of neuropsychological assessment in the presurgical evaluation and postsurgical follow-up of patients with medically refractory temporal lobe epilepsy. In: Radhakrishnan K. editor Medically Refractory Epilepsy. Trivandrum, India: Sree Chitra Tirunal Institute for Medical Sciences and Technology; 1999. p. 159-63.

22. Elizabeth J, Bhaskara RM, Radhakrishnan VV, Radhakrishnan K, Thomas SV. Melanotic differentiation in dysembryoplastic neuroepithelial tumor. Clin Neuropathol 2000;19:38-40.

23. Luyken C, Blümcke I, Fimmers R, Urbach H, Elger CE, Wiestler OD, et al. The spectrum of long-term epilepsy-associated tumors: Long-term seizure and tumor outcome and neurosurgical aspects. Epilepsia 2003;44:822-30.

24. Britton JW, Cascino GD, Sharbrough FW, Kelly P.J. Low grade glial neoplasms and intractable partial epilepsy. Efficacy of surgical treatment. Epilepsia 1994;35:1130-5.

25. Cascino GD, Kelly PJ, Sharbrough FW, Hulihan JF, Hirschorn KA, Trenerry MR. Long-term follow-up of stereotactic lesionectomy in partial epilepsy: Predictive factors and electroencephalographic results. Epilepsia 1992;33:639-44.

26. Jennum P, Dhuna A, Davies K, Fiol M, Maxwell R. Outcome of resective surgery for intractable partial epilepsy guided by subdural electrode arrays. Acta Neurol Scand 1993;87:434-7.

27. Packer RJ, Sutton LN, Patel KM, Duhaime AC, Schiff S, Weinstein SR, et al Seizure control following tumor surgery for childhood cortical low-grade gliomas. J Neurosurg 1994;80:998-1002.

28. Kirkpatrick PJ, Honavar M, Janota I, Polkey CE. Control of temporal lobe epilepsy following en bloc resection of low-grade tumors. J Neurosurg $1993 ; 78: 19-25$

29. Schaller B, Rüegg S.J. Brain tumor and seizures: Pathophysiology and its implications for treatment revisited. Epilepsia 2003;44:1223-32.

30. Garcia PA, Barbaro NM, Laxer KD. The prognostic value of postoperative seizures following epilepsy surgery. Neurology 1991;41:1511-2.

31. Radhakrishnan K, So EL, Silbert PL, Cascino GD, Marsh WR, Cha RH, et al. Prognostic implications of seizure recurrence in the first year after anterior temporal lobectomy. Epilepsia 2003;44:77-80.

32. Hamer HM, Najm I, Mohamed A, Wyllie E. Interictal epileptiform discharges in temporal lobe epilepsy due to hippocampal sclerosis versus mesial temporal lobe tumors. Epilepsia 1999;40:1261-8.

33. Pilcher WH, Silbergeld DL, Berger MS, Ojemann GA. Intraoperative electrocorticography during tumor resection: Impact on seizure outcome in patients with gangliogliomas. J Neurosurg 1993;78:891-902.

34. Taylor DC, Falconer MD. Clinical, socioeconomic and psychological changes after temporal lobectomy for epilepsy. Br J Psychiatry 1968;114:1247-61.

35. Mace CJ, Trimble MR. Psychosis following temporal lobe surgery: A report of six cases. .J Neurol Neurosurg Psychiatry 1991;54:639-44.

36. Leinonen E, Tuunainen A, Lepola U. Postoperative psychosis in epileptic patients after temporal lobectomy. Acta Neurol Scand 1994;90:394-9.

37. Fried I, Kim JH, Spencer DD. Limbic and neocortical gliomas associated with seizures: A distinct clinicopathological group. Neurosurg 1994;34:815-23.

Accepted on 05.11.2004.

\section{Invited Comments}

Epilepsy today is an eminently treatable condition in nearly $70-80 \%$ patients. However, there is a small but significant $20-25 \%$ who continue to have seizures, in spite of appropriate and adequate antiepileptic drug therapy. Surgical treatment is a very good option for people with medically refractory temporal lobe epilepsy, which is gaining ground and being practiced worldwide. Though surgical results have been very encouraging we still need to fine-tune the selection criteria to improve the results further and ask the question why every patient is not benefited? As a corollary, maybe there is a need to identify the factors to reject the surgical option.

MRI has greatly contributed to the selection of cases by de- 
lineating structural abnormalities, largely hippocompal atrophy. It stands to reason that if a lesion is responsible for medically refractory temporal lobe epilepsy lesionectomy should give excellent results. This article focuses on a subtype of a structural temporal lobe lesion i.e. tumoral lesion. Seizure freedom was achieved in nearly $80 \%$ of the patients, one-third of them without antiepileptic drugs and $75 \%$ seizure reduction in $90 \%$ of the patients. If the tumor is involving the mesial temporal lobe structures, these too need to be resected. Incomplete tumor resection is an important cause for continued seizures in the postoperative period. It would have been worthwhile to have done postoperative MRI in all the cases (economically difficult proposition!) to know how many had residual tumor, but yet were free from seizures. From the clinical point of view the most important observation is the seizure-free period during the first postoperative year, which portends excellent long-term prognosis.

\section{H. V. Srinivas}

Bangalore, India. E-mail: ieablr@vsnl.net 